\title{
Message From Your New Editor
}

\author{
Raymond M. Klein
}

Department of Psychology and Neuroscience, Faculty of Science, Dalhousie University, Halifax, Canada

In the first sentence of his landmark book, The Organization of Behavior: A Neurospychological Theory, Donald Hebb (1949, p. xi) wrote: "It might be argued that the task of the psychologist, the task of understanding behavior and reducing the vagaries of human thought to a mechanical process of cause and effect, is a more difficult one than that of any other scientist." Among the various methods for achieving this challenging goal is the (psychology) experiment. In his seminal monograph, Memory. A Contribution to Experimental Psychology Hermann Ebbinghaus provides the reader with this description: "We all know of what this method consists: an attempt is made to keep constant the mass of conditions which have proven themselves causally connected with a certain result; one of these conditions is isolated from the rest and varied in a way that can be numerically described; then the accompanying change on the side of the effect is ascertained by measurement or computation" (1885/1964, p. 7). And his ingenious use of this method in exploring his own behavior allowed Ebbinghaus to discover enduring and general principles about memory.

I received my inculcation into the methods of experimental psychology initially (as an undergraduate) from Marvin Levine (concept formation) and Roger Schvanaveldt (semantic priming) and later (as a graduate student) from Steven Keele (motor control) and Michael Posner (attention). As a young professor at Dalhousie University I was exposed to the design and use of experiments in animal learning, physiological psychology (which we now call behavioral and cognitive neuroscience), and developmental psychology. And more recently in clinical and social psychology. As such, experimental psychology is not so much a topic area that psychologists study as it is a method we can use regardless of our topic area. This methodological focus and topical breadth characterizes the international journal, Experimental Psychology. I am honoured and proud to be appointed its Editor-in-Chief, and excited by the opportunity to lead the journal.

It is a new editor's prerogative, indeed, responsibility, to provide the journal's readership, with a clear picture of their vision for the direction of the journal. In this regard, I am pleased to inform you (though you probably know this already) that the previous editors, Andreas Eder and Christian Frings, and Christoph Stahl before them, have set the journal on a sound course. It is my intention to stay the course.

Eder and Frings (2018) titled their 2018 editorial, What makes a quality journal? They provided 5 answers to their editorial's titular question. The answers were and are, briefly: A good journal has a specialty, has rigorous peerreview, is transparent, values reproducible data and is author friendly. Without repeating their narrative descriptions, let me say that I completely agree with these guiding principles and that as editor I intend to ensure that Experimental Psychology will continue to adhere to them.

The dissemination of scientific knowledge generally, and of particular relevance to us, the dissemination of psychological scientific knowledge, is in a period of flux. Reflecting this journal's leadership in the transition to a more transparent and open scientific process, Experimental Psychology: introduced the registered report in 2014; introduced registered replications (see: https:// www.hogrefe.com/us/journals/exppsy/call-for-replicationstudies), with the possibility of a $€ 500$ grant, in 2020; and was one of the first signatories of the Transparency and Openness Promotions (TOP) guidelines (Nosek et al., 2015). As described in the outgoing editors' most recent editorial (Eder \& Frings, 2021), Experimental Psychology became an early adopter of the Peer-Community In Registered Reports - or in short: PCI RR (https://rr. peercommunityin.org/about/about). It is encouraging that early evidence suggests that research quality is improving because of trends like these (Soderberg et al., 2021).

Experimental Psychology is in the psychology/psychiatry family of journals published by Hogrefe Publishing. I recently attended the first meeting of Hogrefe editors (virtually due to the pandemic). Illustrating Hogrefe's commitment to open publishing, the theme of this inaugural conference was: Together on the road towards open access. In future editorials I expect that I will have more to say about this transition.

As I take the helm (along with the journal's new editorial assistant, Swasti Arora), let me thank the previous 
Editors-in-Chief, Andreas Eder and Christian Frings and their editorial assistants, Anand Krishna and Sarah Schäfer for their groundwork with the journal during their term and for their help and guidance during the editorship changeover. I am also grateful that six members of the previous team of Associate Editors (Matthias Wieser, Alexander Schütz, James Schmidt, Manuel Perea, Gesa Hartwigsen, and Ullrich Ecker) have agreed to continue in this role. Thanks also to the four Associate Editors (Julia Vogt, Sara Teige-Mocigemba, Samual Shaki, and Jörg Rieskamp) who have retired from this role during the last year.

Let me conclude by inviting you to send us your best experimental psychology original reports, registrations, registered replications, and/or theoretical articles.

\section{References}

Ebbinghaus, H. (1885/1964). Memory: A contribution to experimental psychology. Dover.

Eder, A. B., \& Frings, C. (2018). What makes a quality journal? Experimental Psychology, 65(5), 257-262. https://doi.org/10. 1027/1618-3169/a000426
Eder, A. B., \& Frings, C. (2021). Registered report 2.0: The PCI RR initiative. Experimental Psychology, 68(1), 1-3. https://doi.org/10. 1027/1618-3169/a000510

Hebb, D. O. (1949). The organization of behavior: A neuropsychological theory. Wiley.

Nosek, B. A., Alter, G., Banks, G. C., Borsboom, D., Bowman, S. D., Breckler, S. J., Buck, S., Chambers, C. D., Chin, G., Christensen, G., Contestabile, M., Dafoe, A., Eich, E., Freese, J., Glennerster, R., Goroff, D., Green, D. P., Hesse, B., Humphreys, M., .. Yarkoni, T. (2015). Promoting an open research culture. Science, 348(6242), 1422-1425. https://doi.org/10.1126/science. aab2374

Soderberg, C. K., Errington, T. M., Schiavone, S. R., Bottesini, J., Thorn, F. S., Vazire, S., ... \& Nosek, B. A. (2021). Initial evidence of research quality of registered reports compared with the standard publishing model. Nature Human Behaviour, 5, 990-997. https://doi.org/10.1038/s41562-021-01142-4

Published online September 2, 2021

Raymond M. Klein

Department of Psychology and Neuroscience

Faculty of Science

Dalhousie University

355 Oxford St.

Halifax, Nova Scotia B3H 4R2

Canada

ray.klein@dal.ca 